\title{
Sub-Nyquist MIMO Radar Prototype with Doppler Processing
}

David Cohen, Kumar Vijay Mishra, Deborah Cohen, Eran Ronen, Yana Grimovich, Moshe Namer, Maxim Meltsin, and Yonina C. Eldar

\begin{abstract}
We present a hardware prototype of the sub-Nyquist multiple input multiple output radar (SUMMeR) system with capabilities of cognitive transmission and Doppler processing. The prototype allows both spatial and spectral compressive sampling. It realizes an $\mathrm{X}$-band MIMO radar receiver configured to have a maximum of 8 transmit and 10 receive antenna elements. The orthogonality of MIMO waveforms is achieved via frequency division multiplexing. Targets range, azimuth and Doppler are recovered using the Xampling framework. The thinned 4x5 random array configuration achieves the detection performance of its filled array counterpart with samples obtained at $12.5 \%$ of Nyquist from half the antennas.
\end{abstract}

Keywords-MIMO radar, sub-Nyquist, compressed sensing, collocated, cognitive radar

\section{INTRODUCTION}

In recent years, multiple input multiple output (MIMO) radar has become an active area of research [1]. A MIMO radar uses an array of several transmit and receive antenna elements, with each transmitter radiating a different, mutually orthogonal waveform. The waveform orthogonality can be in time, frequency or code. Our system is based on the collocated MIMO configuration [2], in which the elements are close to each other so that the radar cross-section of a target appears identical to all the elements. The MIMO receiver separates and coherently processes the target echoes corresponding to each transmitter. The angular resolution of a MIMO system using the classic virtual ULA is the same as a phased array with equivalent virtual aperture but many more antenna elements than MIMO.

Conventional MIMO radar's spatial (angular) and range resolutions are limited by the number of elements and the receiver sampling rate, respectively. Several methods have been proposed to address the problem of preserving the MIMO radar resolution when either the number of antennas [3] or the number of received samples $[4,5]$ is reduced. These systems exploit the fact that the target scene is sparse, thereby allowing the use of compressed sensing (CS) techniques $[6,7]$.

In [8], a sub-Nyquist collocated MIMO radar (SUMMeR) was proposed to recover the target range, azimuth and Doppler velocity by simultaneously thinning an antenna array and sampling received signals at sub-Nyquist rates. The recovery algorithm uses the Xampling framework where Fourier coefficients of the received signal are acquired from their low-rate samples (or Xamples) [7, 9]. Application of Xampling in space and time enables sub-Nyquist sampling without loss of any of the aforementioned radar resolutions. In SUMMeR, the radar antenna elements are randomly placed within the aperture, and signal orthogonality is achieved by frequency division multiplexing (FDM). The FDM-based sub-Nyquist MIMO

This project has received funding from the European Union's Horizon 2020 research and innovation programme under grant agreement No. 646804-ERC-COG-BNYQ. K.V.M. acknowledges partial support via Andrew and Erna Finci Viterbi Fellowship. D.C. is grateful to the Azrieli Foundation for the award of an Azrieli Fellowship. mitigates the range-azimuth coupling by randomizing the element locations in the aperture.

Our very recent work [10] presents a hardware prototype of the SUMMeR system and demonstrates its feasibility via real-time experiments. This implementation follows the recommendations of [11] for signal orthogonality, array structure and reconstruction algorithms. There, only the targets' azimuth and range are recovered, from a single pulse per transmitter. The prototype can be configured either as a filled or thinned array, thereby allowing comparison of Nyquist and sub-Nyquist spatial sampling using the same hardware.

In this work, we extend the SUMMeR prototype to Doppler processing and simultaneously recover all three parameters, range, azimuth and Doppler, in real-time. Additionally, we use cognitive transmission techniques [12], as in [10], wherein the transmit signal is limited to those selected subbands that a sub-Nyquist receiver samples and processes. In one of our previous works on single antenna radar systems [13], the sub-Nyquist radar hardware pre-filters a few randomly chosen, narrow subbands of the received signal spectrum before sampling them using low-rate analog-to-digital converters (ADCs). The transmit signal is, however, full-band even though only a few subbands are sampled and processed by the receiver. Cognitive transmission provides two advantages to SUMMeR hardware. First, the spatial sub-Nyquist processing of large arrays can be easily designed without replicating the pre-filtering operation for each subband in the hardware. Second, since the total transmit power remains the same, a cognitive signal has more in-band power resulting in an increase in signal-to-noise ratio (SNR).

In the following section, we review the theory of SUMMeR including the recovery algorithm for range, azimuth and Doppler. We then describe the design of our prototype and its major features in Section III, and present results obtained by the prototype in real-time experiments.

\section{Sub-Nyquist Collocated MIMO Radar}

With the exception of cognitive transmission, the array and signal models of SUMMeR realized by our prototype closely follow that detailed by [8] and are only summarized her.

\section{A. MIMO Radar Model}

Let the operating wavelength of the radar be $\lambda$ and the total number of transmit and receive elements be $T$ and $R$ respectively. The classic approach to collocated MIMO adopts a virtual uniform linear array (ULA) structure [14], where the receive antennas spaced by $\frac{\lambda}{2}$ and transmit antennas spaced by $R \frac{\lambda}{2}$ form two ULAs (or vice versa, see e.g. [15]). Here, the coherent processing of a total of $T R$ channels in the receiver creates a virtual equivalent of a phased array with $T R \frac{\lambda}{2}$-spaced receivers and normalized aperture $Z=\frac{T R}{2}$.

Consider a collocated MIMO radar system that has $M<T$ transmit and $Q<R$ receive antennas. The locations of these antennas 
are chosen uniformly at random within the aperture of the virtual array mentioned above. The $m$ th transmitting antenna sends $P$ pulses $s_{m}(t)$ given by

$$
s_{m}(t)=\sum_{p=0}^{P-1} h_{m}(t-p \tau) e^{j 2 \pi f_{c} t}, \quad 0 \leq t \leq P \tau,
$$

where $\tau$ denotes the pulse repetition interval (PRI), $P \tau$ is the coherent processing interval (CPI), $f_{c}$ is the common carrier frequency at the radio frequency (RF) stage, and $\left\{h_{m}(t)\right\}_{m=0}^{M-1}$ is a set of narrowband, orthogonal FDM pulses each with the continuous-time Fourier transform (CTFT)

$$
H_{m}(\omega)=\int_{-\infty}^{\infty} h_{m}(t) e^{-j \omega t} d t
$$

For simplicity, we assume that $f_{c} \tau$ is an integer, so that the initial phase for every pulse $e^{-j 2 \pi f_{c} \tau p}$ is canceled in the modulation [16]. The pulse time support is denoted by $T_{p}$.

Consider a target scene with $L$ non-fluctuating point targets following the Swerling-0 model [17] whose locations are given by their ranges $R_{l}$, Doppler velocity $v_{l}$, and azimuth angles $\theta_{l}, 1 \leq l \leq L$. The pulses transmitted by the radar are reflected back by the targets and collected at the receive antennas. When the received waveform is downconverted from RF to baseband, we obtain the following signal at the $q$ th antenna,

$$
x_{q}(t)=\sum_{p=0}^{P-1} \sum_{m=0}^{M-1} \sum_{l=1}^{L} \alpha_{l} h_{m}\left(t-p \tau-\tau_{l}\right) e^{j 2 \pi \beta_{m q} \vartheta_{l}} e^{j 2 \pi f_{l}^{D} p \tau},
$$

where $\alpha_{l}$ denotes the complex-valued reflectivity of the $l$ th target, $c$ is the speed of light, $\tau_{l}=2 R_{l} / c$ is the range-time delay the $l$ th target, $f_{l}^{D}=\frac{2 v_{l}}{c} f_{c}$ is the frequency in the Doppler spectrum, $\vartheta_{l}=\sin \theta_{l}$ is the azimuth parameter, and $\beta_{m q}$ is governed by the array structure. It will be convenient to express $x_{q}(t)$ as a sum of single frames

$$
x_{q}(t)=\sum_{p=0}^{P-1} x_{q}^{p}(t)
$$

where

$$
x_{q}^{p}(t)=\sum_{m=0}^{M-1} \sum_{l=1}^{L} \alpha_{l} h\left(t-\tau_{l}-p \tau\right) e^{j 2 \pi \beta_{m q} \vartheta_{l}} e^{j 2 \pi f_{l}^{D} p \tau} .
$$

Our goal is to estimate the time delay $\tau_{l}$, azimuth $\theta_{l}$, and Doppler shifts $f_{l}^{D}$ of each target from low rate samples of $x_{q}(t)$, for $0 \leq q \leq$ $Q-1$, and a small number of $M$ channels and $Q$ antennas.

\section{B. Xampling in Time and Space}

The application of Xampling in both space and time enables recovery of range, direction and velocity at sub-Nyquist rates. The performance guarantees of this procedure are provided in [8]. The received signal $x_{q}(t)$ is separated into $M$ channels, aligned and normalized. The Fourier coefficients of the received signal corresponding to the channel that processes the $m$ th transmitter echo at the $q$ th receiver are given by

$$
y_{m, q}^{p}[k]=\sum_{l=1}^{L} \alpha_{l} e^{j 2 \pi \beta_{m q} \vartheta_{l}} e^{-j \frac{2 \pi}{\tau} k \tau_{l}} e^{-j 2 \pi f_{m} \tau_{l}} e^{j 2 \pi f_{l}^{D} p \tau},
$$

where $-\frac{N}{2} \leq k \leq-\frac{N}{2}-1, f_{m}$ is the (baseband) carrier frequency of the $m$ th transmitter and $N$ is the number of Fourier coefficients per channel. Xampling obtains a set $\kappa$ of arbitrarily chosen Fourier coefficients from low rate samples of the received channel signal such that $|\kappa|=K<N$. More details can be found in [13].
As in traditional MIMO, assume that the time delays, azimuths and Doppler frequencies are aligned to a grid. In particular, $\tau_{l}=\frac{\tau}{T N} s_{l}$, $\vartheta_{l}=-1+\frac{2}{T R} r_{l}$ and $f_{l}^{D}=-\frac{1}{2 \tau}+\frac{1}{P \tau} u_{l}$, where $s_{l}, r_{l}$ and $u_{l}$ are integers satisfying $0 \leq s_{l} \leq T N-1,0 \leq r_{l} \leq T R-1$ and $0 \leq u_{l} \leq P-1$, respectively. Let $\mathbf{Z}^{m}$ be the $K Q \times P$ matrix with $q$ th column given by the vertical concatenation of $y_{m, q}^{p}[k], k \in \kappa$, for $0 \leq q \leq Q-1$. We can then write $\mathbf{Z}^{m}$ as

$$
\mathbf{Z}^{m}=\left(\overline{\mathbf{B}}^{m} \otimes \mathbf{A}^{m}\right) \mathbf{X}_{D} \mathbf{F}^{H} .
$$

Here, $\mathbf{A}^{m}$ denotes the $K \times T N$ matrix whose $(k, n)$ th element is $e^{-j \frac{2 \pi}{T N} \kappa_{k} n} e^{-j 2 \pi \frac{f_{m}}{B_{h}} \frac{n}{T}}$ with $\kappa_{k}$ the $k$ th element in $\kappa, \mathbf{B}^{m}$ is the $Q \times T R$ matrix with $(q, p)$ th element $e^{-j 2 \pi \beta_{m q}\left(-1+\frac{2}{T R} p\right)}$ and $\mathbf{F}$ denotes the $P \times P$ Fourier matrix. The Kronecker product is denoted by $\otimes$ and $(\cdot)^{H}$ is the Hermitian operator. The matrix $\mathbf{X}_{D}$ is a $T^{2} N R \times P$ sparse matrix that contains the values $\alpha_{l}$ at the $L$ indices $\left(r_{l} T N+s_{l}, u_{l}\right)$.

Our goal is now to recover $\mathbf{X}_{D}$ from the measurement matrices $\mathbf{Z}^{m}, 0 \leq m \leq M-1$. The time, spatial and frequency resolution stipulated by $\mathbf{X}_{D}$ are $\frac{1}{T B_{h}}, \frac{2}{T R}$ and $\frac{1}{P \tau}$ respectively. Theorem 1 presents necessary conditions on the minimal number of channels $M Q$, samples per receiver $M K$ and pulses per transmitter $P$ for perfect recovery of $\mathbf{X}_{D}$ from (7) under the grid assumption. The proof can be found in [8].

Theorem 1. The minimal number of channels required for perfect recovery of $\mathbf{X}_{D}$ with $L$ targets in noiseless settings is $M Q \geq 2 L$ with a minimal number of $M K \geq 2 L$ samples per receiver and $P \geq 2 L$ pulses per transmitter.

\section{Range-Azimuth-Doppler Recovery}

To jointly recover the range, azimuth and Doppler frequency of the targets, we apply the concept of Doppler focusing from [9] to our MIMO setting. Once the Fourier coefficients are acquired and processed, we perform Doppler focusing for a specific frequency $v$, that is

$$
\begin{aligned}
\Phi_{m, q}^{v}[k] & =\sum_{p=0}^{P-1} y_{m, q}^{p}[k] e^{-j 2 \pi v p \tau} \\
& =\sum_{l=1}^{L} \alpha_{l} e^{j 2 \pi \beta_{m q} \vartheta_{l}} e^{-j \frac{2 \pi}{\tau}\left(k+f_{m} \tau\right) \tau_{l}} \sum_{p=0}^{P-1} e^{j 2 \pi\left(f_{l}^{D}-v\right) p \tau},
\end{aligned}
$$

for $-\frac{N}{2} \leq k \leq-\frac{N}{2}-1$. Following [9], it holds that

$$
\sum_{p=0}^{P-1} e^{j 2 \pi\left(f_{l}^{D}-v\right) p \tau} \cong \begin{cases}P & \left|f_{l}^{D}-v\right|<\frac{1}{2 P \tau}, \\ 0 & \text { otherwise. }\end{cases}
$$

Then, for each focused frequency $v,(8)$ reduces to a 2-dimensional problem. The system (7) can be solved using CS recovery techniques, such as orthogonal matching pursuit (OMP), which is extended to simultaneous 3-dimensional sparse recovery with Doppler focusing [8].

Algorithm 1 solves (7) using Doppler focusing. Note that step 1 can be performed using fast Fourier transform (FFT). In the algorithm description, $\operatorname{vec}(\mathbf{Z})$ concatenates the columns of $\mathbf{Z}^{m}$, for $0 \leq m \leq$ $M-1, \mathbf{e}_{t}(l)=\left[\left(\mathbf{e}_{t}^{0}(l)\right)^{T} \cdots\left(\mathbf{e}_{t}^{M-1}(l)\right)^{T}\right]^{T}$ where $\mathbf{e}_{t}^{m}(l)=\operatorname{vec}\left(\left(\overline{\mathbf{B}}^{m} \otimes\right.\right.$ $\left.\left.\mathbf{A}^{m}\right)_{\Lambda_{t}(l, 2) T N+\Lambda_{t}(l, 1)}\left(\left(\overline{\mathbf{F}}^{m}\right)_{\Lambda_{t}(l, 3)}^{T}\right)^{T}\right)$ with $\Lambda_{t}(l, i)$ the $(l, i)$ th element in the index set $\Lambda_{t}$ at the $t$ th iteration, and $\mathbf{E}_{t}=\left[\mathbf{e}_{t}(1) \ldots \mathbf{e}_{t}(t)\right]$. Once $\mathbf{X}_{D}$ is recovered, the delays, azimuths and Dopplers are estimated as

$$
\hat{\tau}_{l}=\frac{\tau \Lambda_{L}(l, 1)}{T N}, \hat{\vartheta}_{l}=-1+\frac{2 \Lambda_{L}(l, 2)}{T R}, \text { hat }_{l}^{D}=-\frac{1}{2 \tau}+\frac{\Delta_{L}(l, 3)}{P \tau} .
$$


Since in real scenarios, targets delays, Dopplers and azimuths are not necessarily aligned to a grid, a finer grid can be used around detection points on the coarse grid to reduce quantization error. This technique, we refer to as dynamic grid, simply adds a step after support detection in each iteration (step 4 in Algorithm 1), that refines the grid around the detected azimuth, range and Doppler frequency.

Algorithm 1 Simultaneous sparse 3D recovery based OMP with focusing

Input: Observation matrices $\mathbf{Z}^{m}$, measurement matrices $\mathbf{A}^{m}, \mathbf{B}^{m}$, for all $0 \leq m \leq M-1$

Output: Index set $\Lambda$ containing the locations of the non zero indices of $\mathbf{X}$, estimate for sparse matrix $\hat{\mathbf{X}}$

1: Perform Doppler focusing for $0 \leq i \leq K-1$ and $0 \leq j \leq Q-1$ :

$$
\boldsymbol{\Phi}_{i, j}^{(m, v)}=\sum_{p=0}^{P-1} \mathbf{Z}_{i+j K, p}^{m} e^{j 2 \pi v p \tau} .
$$

2: Initialization: residual $\mathbf{R}_{0}^{(m, v)}=\boldsymbol{\Phi}^{(m, v)}$, index set $\Lambda_{0}=\emptyset, t=1$

3: Project residual onto measurement matrices for $0 \leq p \leq P-1$ :

$$
\boldsymbol{\Psi}^{v}=\mathbf{A}^{H} \mathbf{R}^{v} \mathbf{B},
$$

where $\mathbf{A}=\left[\begin{array}{lll}\mathbf{A}^{0^{T}} & \mathbf{A}^{1^{T}} \cdots \mathbf{A}^{(M-1)^{T}}\end{array}\right]^{T}, \mathbf{B}=\left[\mathbf{B}^{0^{T}} \mathbf{B}^{1^{T}} \cdots \mathbf{B}^{(M-1)^{T}}\right]^{T}$ and $\mathbf{R}^{v}=\operatorname{diag}\left(\left[\mathbf{R}_{t-1}^{(0, v)} \cdots \mathbf{R}_{t-1}^{(M-1, v)}\right]\right)$ is block diagonal

4: Find the three indices $\lambda_{t}=\left[\lambda_{t}(1) \lambda_{t}(2) \lambda_{t}(3)\right]$ such that

$$
\left[\begin{array}{lll}
\lambda_{t}(1) & \lambda_{t}(2) & \lambda_{t}(3)
\end{array}\right]=\arg \max _{i, j, v}\left|\Psi_{i, j}^{v}\right|
$$

5: Augment index set $\Lambda_{t}=\Lambda_{t} \cup\left\{\lambda_{t}\right\}$

6: Find the new signal estimate

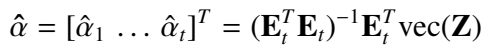

7: Compute new residual

$$
\mathbf{R}_{t}^{(m, v)}=\mathbf{Z}^{m}-\sum_{l=1}^{t} \alpha_{l} e^{j 2 \pi\left(-\frac{1}{2}+\frac{\Lambda_{t}(l, 3)}{P}\right) p} \mathbf{a}_{\Lambda_{t}(l, 1)}^{m}\left(\overline{\mathbf{b}}_{\Lambda_{t}(l, 2)}^{m}\right)^{T}
$$

8: If $t<L$, increment $t$ and return to step 2, otherwise stop

9: Estimated support set $\hat{\Lambda}=\Lambda_{L}$

10: Estimated matrix $\hat{\mathbf{X}}_{D}:\left(\Lambda_{L}(l, 2) T N+\Lambda_{L}(l, 1), \Lambda_{L}(l, 3)\right)$-th component is given by $\hat{\alpha}_{l}$ while rest of the elements are zero

\section{Cognitive Transmission}

Let $\mathcal{B}_{m}$ be the set of all frequencies in the $m$ th transmit signal spectrum of effective bandwidth $B_{h}$. In the cognitive radar transmission, the spectrum $\tilde{H}_{m}(\omega)$ of each of the transmitted waveforms $\tilde{h}_{m}(t)$ is limited to a total of $N_{b}$ non-overlapping frequency bands $\mathcal{B}_{i}, 1 \leq i \leq N_{b}$ :

$$
\tilde{H}_{m}(\omega)=\left\{\begin{array}{l}
\gamma(\omega) H_{m}(\omega), \quad \omega \in \bigcup_{i=1}^{N_{b}} \mathcal{B}_{i} \subset \mathcal{B}_{h} \\
0, \quad \text { otherwise }
\end{array}\right.
$$

where $\gamma(\omega)=B_{h} /\left|\mathcal{B}_{i}\right|$ for $\omega \in \mathcal{B}_{i}$. The total transmit power $P_{t}$ remains the same [18] such that the power relation between the conventional and cognitive waveforms is

$$
\int_{-B_{h} / 2}^{B_{h} / 2}\left|H_{m}(\omega)\right|^{2} \mathrm{~d} \omega=\sum_{i=1}^{N_{b}} \int_{\mathcal{B}_{i}}\left|\tilde{H}_{m}(\omega)\right|^{2} \mathrm{~d} \omega=P_{t}
$$

In a cognitive radar, the sub-Nyquist receiver obtains the set $\kappa$ of the Fourier coefficients only from the subbands $\mathcal{B}_{i}$.
TABLE I. TEChNICAL CHARACTERISTICS OF THE PROTOTYPE

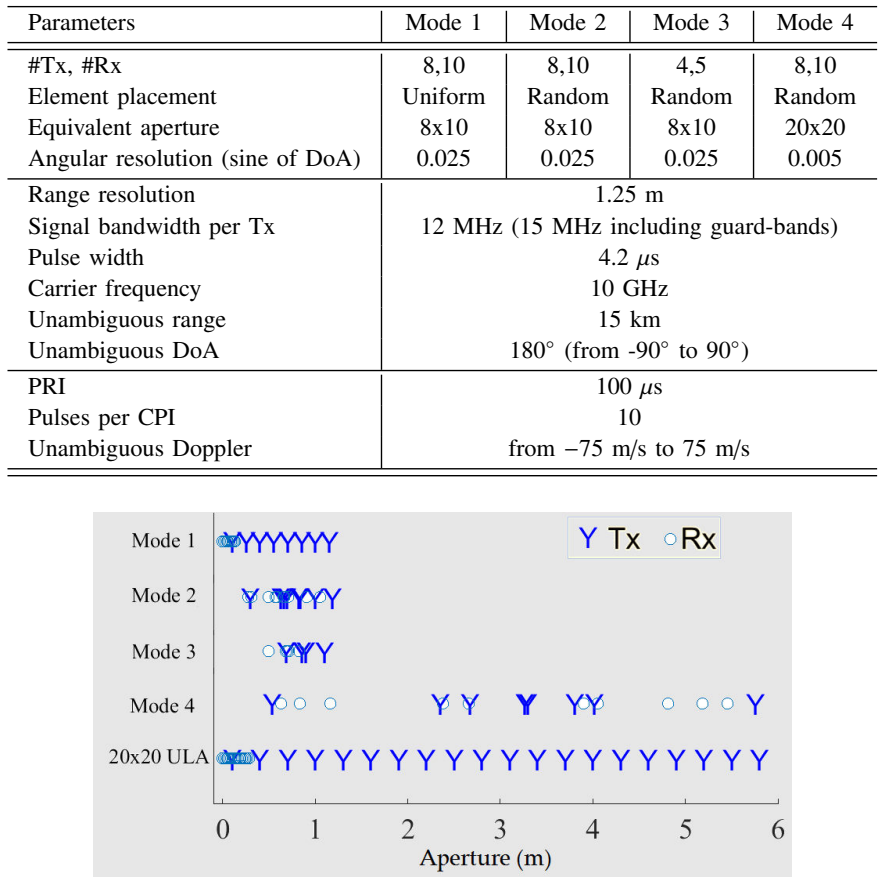

Fig. 1. Tx and Rx element locations for the hardware prototype modes over a $6 \mathrm{~m}$ antenna aperture. Mode 4's virtual array equivalent is the $20 \times 20$ ULA.

\section{Hardware Prototype ANd Experiments}

The cognitive SUMMeR prototype realizes a receiver with a maximum of 8 transmit (Tx) and 10 receive $(\mathrm{Rx})$ antenna elements. The experimental process consists of the following steps. The simulated radar scenario is stored in a custom-designed waveform generator. The scenario includes modeling of pulse transmission, accurate power loss due to wave propagation in a realistic medium, and interaction of transmit signal with the target. A large variety of scenarios, consisting of different target parameters, i.e. delays, Doppler frequencies, and amplitudes, and array configurations, i.e. number of transmitters and receivers and antenna locations, can be examined using the prototype. The waveform generator board then produces an analog signal corresponding to the synthesized radar environment, which is amplified and routed to the MIMO radar receiver board. The prototype then samples and processes the signal in real-time. The physical array aperture and simulated target response correspond to an X-band $\left(f_{c}=10 \mathrm{GHz}\right)$ radar.

Our previous work [10] describes the design philosophy of the prototype. Briefly, we use the same hardware for each receive element and serially feed the signals of all 10 receivers to the same prototype. Further, in order to avoid use of an overwhelmingly large number of ADCs and bandpass filters for an $8 \times 10$ array, we adopt a cognitive transmission wherein each transmit signal lies in $N_{b}=8$ disjoint, narrow slices. More importantly, for each channel, a single low-rate ADC can subsample this narrow-band signal as long as the subbands are coset bands so that they do not alias after sampling [19].

Table I lists details technical characteristics of the prototype can be found in [10]. The system can be configured to operate in various array configurations or modes. Mode 3 and 4 are sub-Nyquist MIMO modes; the hardware switches off the inactive channels and does not 


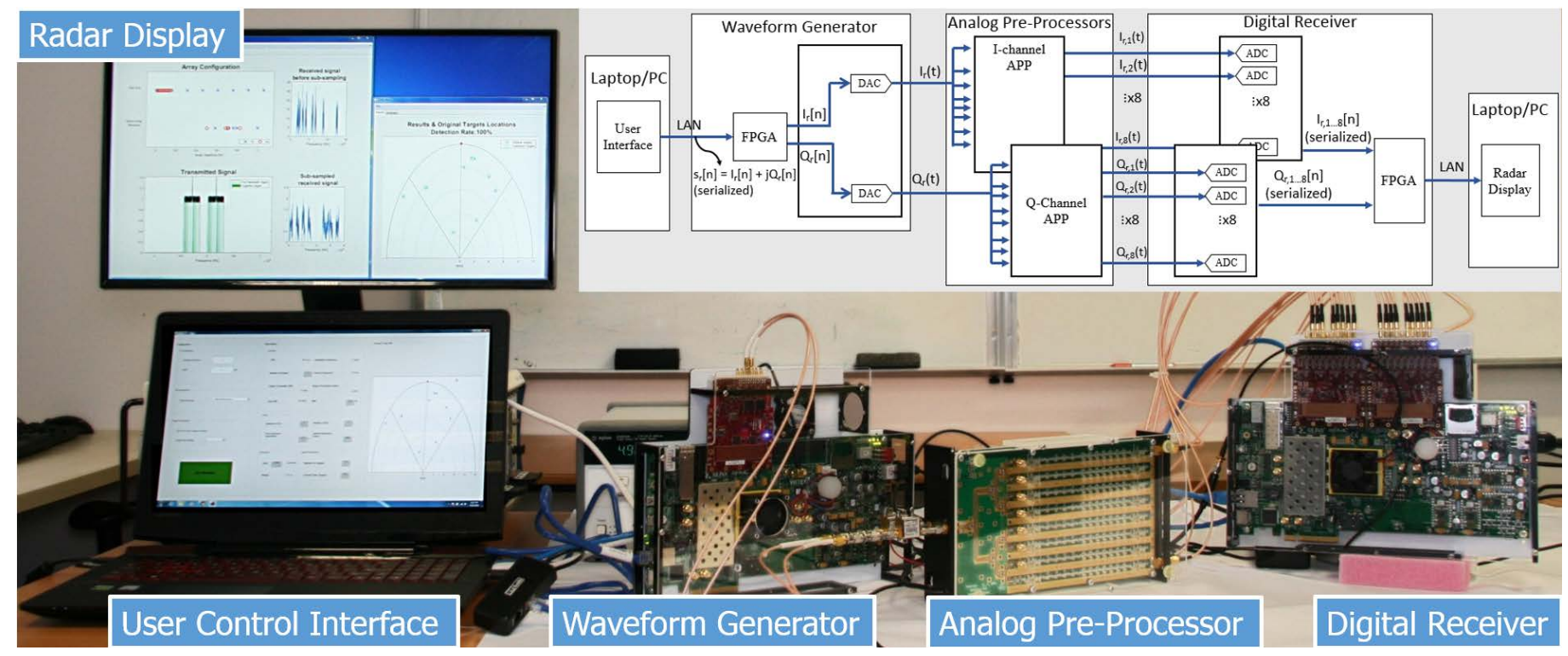

Fig. 2. Sub-Nyquist MIMO prototype and user interface. The analog pre-processor (APP) module consists of two cards mounted on opposite sides of a common chassis. The inset shows the simplified block diagram of the system. The subscript $r$ represents received signal samples for $r$ th receiver. Wherever applicable, the second subscript corresponds to a particular transmitter. The square brackets (parentheses) are used for digital (analog) signals.

sample any data over the corresponding ADCs. Figure 2 shows the sub-Nyquist MIMO prototype, user interface and radar display. The inset graph depicts the signal flow through a simplified block diagram.

As shown in Figure 3a, the cognitive radar signal occupies only certain subbands in a $15 \mathrm{MHz}$ band. Here, the sliced transmit signal has eight subbands each of width $375 \mathrm{kHz}$. with the frequency range of 1.63-2, 2.16-2.53, 3.05-3.42, 3.88-4.25, 5.66-6.03, 6.51-6.88, 8.64-9.01 and $12.32-12.69 \mathrm{MHz}$ before subsampling. The total signal bandwidth is $0.375 \times 8=3 \mathrm{MHz}$. This signal is subsampled at 7.5 $\mathrm{MHz}$ and the subbands locations were chosen so that there is no aliasing between different subbands (Fig. 3b). A non-cognitive signal would have occupied the entire $15 \mathrm{MHz}$ spectrum requiring a Nyquist sampling rate of $30 \mathrm{MHz}$. Therefore, the use of cognitive transmission enables spectral sampling reduction by a factor of $4(=30 \mathrm{MHz} / 7.5$ $\mathrm{MHz}$ ) for each channel. Depending on whether the guard-bands of the non-cognitive transmission are included in the computation or not, the effective signal bandwidth is reduced by a factor of $5(=15 \mathrm{MHz} / 3$ $\mathrm{MHz})$ or $4(=12 \mathrm{MHz} / 3 \mathrm{MHz})$ respectively for each channel. Mode 3 has $50 \%$ spatial sampling reduction when compared with Mode 1 or 2.

We evaluated the performance of all the modes through hardware simulations. In the experiment, $P=10$ pulses were transmitted at a uniform pulse repetition frequency (PRF) of $100 \mu$ s and all modes were evaluated against identical target scenarios. We injected the received signal corresponding to the echoes from $L=10$ targets, placed at arbitrary range and azimuths and with arbitrary velocities, in the transmit waveform generator. In the first experiment, when the angular spacing (in terms of the sine of azimuth) between any two targets was greater than 0.025 and the signal $\mathrm{SNR}=-8 \mathrm{~dB}$, the recovery performance of the thinned $4 \times 5$ array in Mode 3 was not worse than Modes 1 and 2. For this experiment, Figs. 4 and 5 show the plan position indicator (PPI) plot and range-azimuth-Doppler maps of all the modes. Here, a successful detection (green circle) occurs when the estimated target is within one range cell, one azimuth bin and one Doppler bin of the ground truth (blue circle); otherwise, the estimated

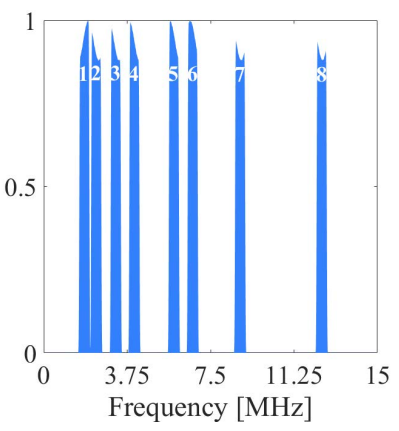

(a) Before subsampling

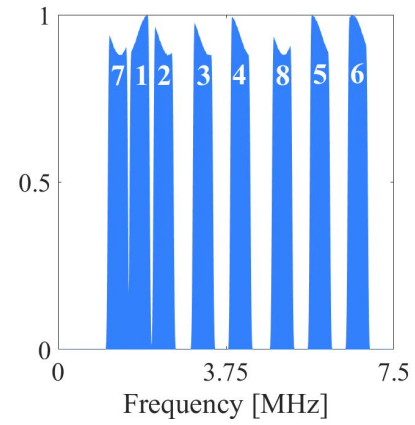

(b) After subsampling
Fig. 3. The normalized one-sided spectrum of one channel of a given receiver (a) before and (b) after subsampling with a $7.5 \mathrm{MHz}$ ADC. Each of the subbands spans $375 \mathrm{kHz}$ and is marked with a numeric label. In a non-cognitive processing, the signal occupies the entire $15 \mathrm{MHz}$ spectrum before sampling.

target is labeled as a false alarm (magenta circle).

We next considered a sparse target scene with $L=10$ targets including two couples of targets with close azimuth dimension, with angular spacing of 0.02 . The SNR of the injected signal was $-5 \mathrm{~dB}$. Since the angular resolution of Mode 4 is better than the other three modes, all the targets are successfully detected in Mode 4. Mode 1 and 3 showed a false alarm as seen in the inset plots of Figs. 6 and 7. Mode 2 also shows successful recovery in the sense of our detection criterion. However, relatively better performance of Mode 2 over Modes 1 and 3 is not entirely fortuitous here. Figure 1 shows that both Tx and Rx array elements in Mode 2 are distributed such that its virtual array is wider than Modes 1 and 3. Thus, the effective angular resolution for Mode 2 could be better than 1 and 3, but still worse than 4 . 


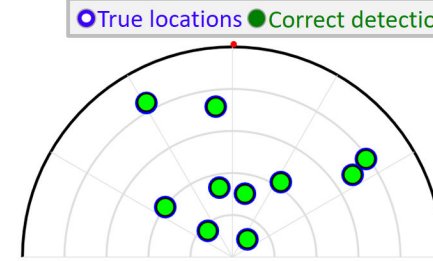

(a) Mode 1 ( 8 × 10 ULA)

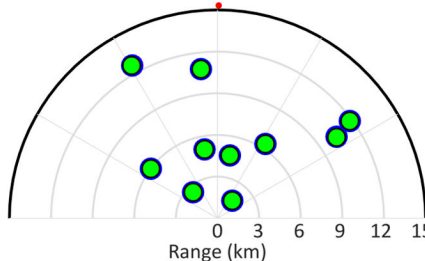

(c) Mode 3 ( $4 \times 5$ thinned)

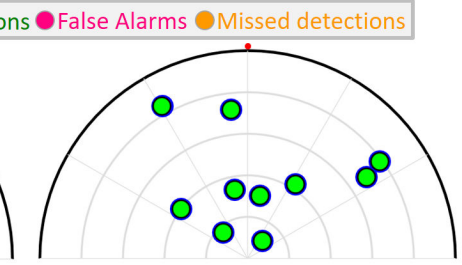

(b) Mode 2 ( $8 \times 10$ filled, random)

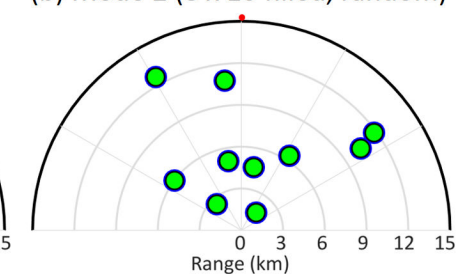

(d) Mode 4 ( $8 \times 10$ thinned)
Fig. 4. Plan Position Indicator (PPI) display of results for Mode 1 and 3 . The origin is the location of the radar. The red dot indicates the north direction relative to the radar. Positive (negative) distances along the horizontal axis correspond to the east (west) of the radar. Similarly, positive (negative) distances along the vertical axis correspond to the north (south) of the radar. The estimated targets are plotted over the ground truth.

\section{OTrue locations Correct detections False Alarms Missed detections}

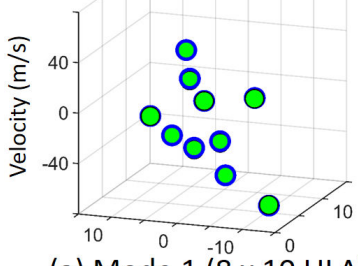

(a) Mode 1 ( $8 \times 10$ ULA)

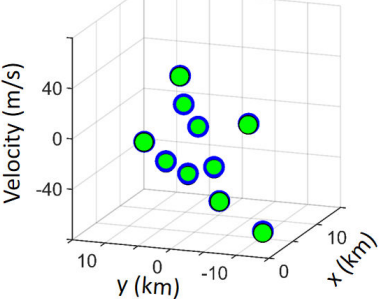

(c) Mode 3 ( $4 \times 5$ thinned)

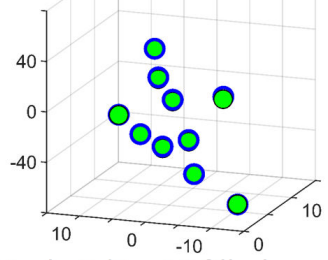

(b) Mode 2 ( 8 × 10 filled, random)

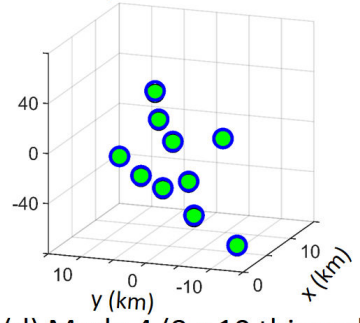

(d) Mode 4 ( 8 × 10 thinned)
Fig. 5. Range-Azimuth-Doppler map for the target configurations shown in Fig. 4. The lower axes represent the Cartesian coordinates of the polar representation of the PPI plots from Fig. 4. The vertical axis represents the Doppler spectrum.

\section{Summary}

We presented the first hardware prototype of sub-Nyquist MIMO that demonstrates real-time operation of spatial reduction in sampling, cognitive transmission and delay-azimuth-Doppler recovery using sub-Nyquist processing. The thinned $4 \times 5$ array has the same detection performance as $8 \times 10$ filled array. The spatial and spectral reduction in the thinned $4 \times 5$ array is $50 \%$ and $87.5 \%$, respectively. The prototype performance is robust with SNRs dropping to as low as $-5 \mathrm{~dB}$. While we did not analyze the performance in the presence of clutter, the prototype design does not restrict such an evaluation and we reserve this implementation for future work.

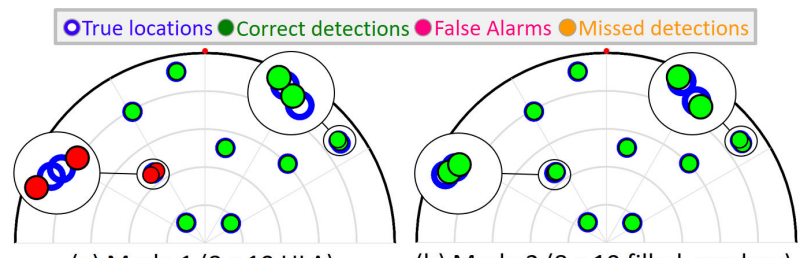

(a) Mode 1 ( 8 x 10 ULA) (c) Mode 3 ( 4 × 5 thinned)

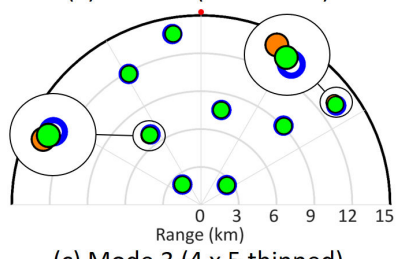

(b) Mode 2 ( $8 \times 10$ filled, random)

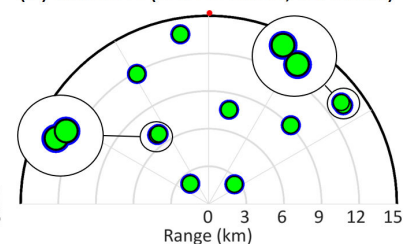

(d) Mode 4 ( $8 \times 10$ thinned)
Fig. 6. As in Fig. 5, but for a closely-spaced target scenario. The inset plots show the selected region in each PPI display on a magnified scale.

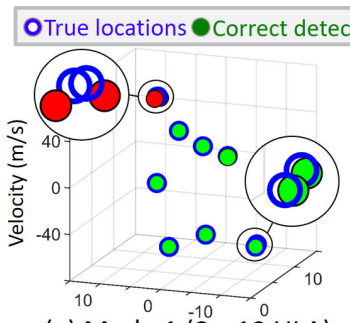

(a) Mode $1(8 \times 10$ ULA)

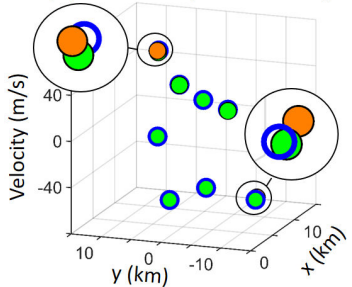

(c) Mode 3 ( $4 \times 5$ thinned)

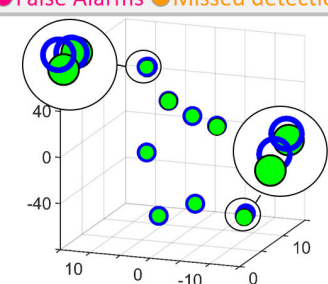

(b) Mode 2 ( $8 \times 10^{-10}$ filled, random)

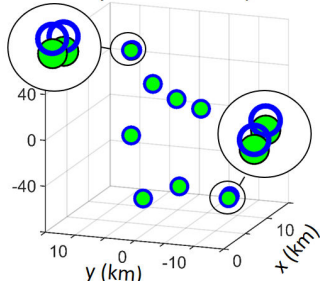

(d) Mode 4 ( $8 \times 10$ thinned)
Fig. 7. As in Fig. 6, but for a closely-spaced target scenario. The inset plots show the selected region in each map on a magnified scale.

\section{REFERENCES}

[1] E. Fishler, A. Haimovich, R. Blum, D. Chizhik, L. Cimini, and R. Valenzuela, "MIMO radar: An idea whose time has come," in IEEE Radar Conf., 2004, pp. 71-78.

[2] J. Li and P. Stoica, "MIMO radar with colocated antennas," IEEE Signal Process. Mag., vol. 24, no. 5, pp. 106-114, 2007.

[3] M. Rossi, A. M. Haimovich, and Y. C. Eldar, "Spatial compressive sensing for MIMO radar," IEEE Trans. Signal Process., vol. 62, no. 2, pp. 419-430, 2014.

[4] Y. Yu, A. P. Petropulu, and H. V. Poor, "MIMO radar using compressive sampling," IEEE J. Sel. Topics in Signal Process., vol. 4, no. 1, pp. 146-163, 2010.

[5] S. Sun, W. U. Bajwa, and A. P. Petropulu, "MIMO-MC radar: A MIMO radar approach based on matrix completion," IEEE Trans. Aerosp. Electron. Syst., vol. 51, no. 3, pp. 1839-1852, 2015.

[6] Y. C. Eldar and G. Kutyniok, Compressed Sensing: Theory and Applications. Cambridge University Press, 2012.

[7] Y. C. Eldar, Sampling Theory: Beyond Bandlimited Systems. Cambridge University Press, 2015.

[8] D. Cohen, D. Cohen, Y. C. Eldar, and A. M. Haimovich, "SUMMeR: Sub-Nyquist MIMO Radar," arXiv preprint arXiv:1608.07799, 2016.

[9] O. Bar-Ilan and Y. C. Eldar, "Sub-Nyquist radar via Doppler focusing," IEEE Trans. on Signal Proc., vol. 62, no. 7, pp. 1796-1811, 2014.

[10] K. V. Mishra, E. Shoshan, M. Namer, M. Meltsin, D. Cohen, 
R. Madmoni, S. Dror, R. Ifraimov, and Y. C. Eldar, "Cognitive Sub-Nyquist hardware prototype of a collocated MIMO radar," in Compressed Sensing Theory and its Applications to Radar, Sonar and Remote Sensing, 2016

[11] D. Cohen, D. Cohen, Y. C. Eldar, and A. M. Haimovich, "Sub-Nyquist collocated MIMO radar in time and space," in IEEE Radar Conf., 2016

[12] D. Cohen, A. Dikopoltsev, R. Ifraimov, and Y. C. Eldar, "Towards sub-Nyquist cognitive radar," in IEEE Radar Conf., 2016.

[13] E. Baransky, G. Itzhak, I. Shmuel, N. Wagner, E. Shoshan, and Y. C. Eldar, "A sub-Nyquist radar prototype: Hardware and applications," IEEE Trans. Aerosp. Electron. Syst., vol. 50, no. 2, pp. 809-822, 2014.

[14] C.-Y. Chen, "Signal processing algorithms for MIMO radar," Ph.D. dissertation, California Institute of Technology, 2009.

[15] E. Brookner, "MIMO radars and their conventional equivalents An update," in IET Int. Radar Conf., 2015, pp. 1-8.

[16] P. Z. Peebles, Radar principles. John Wiley \& Sons, 2007.

[17] M. I. Skolnik, Radar handbook, 3rd ed. McGraw-Hill, 2008.

[18] K. V. Mishra and Y. C. Eldar, "Performance of time delay estimation in a cognitive radar," in IEEE Int. Conf. Acoustics, Speech and Signal Process., 2017, to appear.

[19] K. M. Cohen, C. Attias, B. Farbman, I. Tselniker, and Y. C. Eldar, "Channel estimation in UWB channels using compressed sensing," in IEEE Int. Conf. on Acoustics, Speech and Signal Processing, 2014, pp. 1966-1970. 